\title{
ALL FUNDAMENTAL GROUPS ARE ALMOST CONTACT
}

\section{KOTSCHICK}

It was proved in [2] that in dimension 4, and therefore in any even dimension $\geqslant 4$, all finitely presentable groups arise as fundamental groups of closed almost complex, or, equivalently, almost symplectic manifolds. We prove here the analogous result in odd dimensions.

THEOREM. Every finitely presentable group is the fundamental group of a closed almost contact manifold of dimension 5.

This is a consequence of the result in [2] combined with the following.

Proposition. If a group $\Gamma$ arises as the fundamental group of a closed almost symplectic $2 n$-manifold, $n \geqslant 2$, then it also arises as the fundamental group of a closed almost contact $(2 n+1)$-manifold which is a circle bundle over an almost symplectic manifold with fundamental group $\Gamma$.

If the structure on the base space of the bundle is integrable, then the one on the total space can be chosen to be integrable as well. In this case, our construction reduces to that of Boothby and Wang [1], enhanced by control on the fundamental group, and we obtain an inclusion of the class of fundamental groups of symplectic $2 n$-manifolds into that of fundamental groups of contact $(2 n+1)$-manifolds. This is interesting only for $n=2$, because in dimensions $2 n+1 \geqslant 7$, all finitely presentable groups arise as fundamental groups of contact manifolds which are the unit tangent bundles of closed Riemannian $(n+1)$-manifolds. Note also that all orientable 3 manifolds admit contact structures [3].

Proof of Proposition. Let $X$ be a $2 n$-manifold and $\pi: Y \rightarrow X$ a principal $S^{1}$ bundle. Every connexion on the bundle defines a splitting $T Y=T_{\pi} \oplus \pi^{*} T X$. Hence the structure group of $T Y$ reduces to $\mathrm{U}(n)$ if the structure group of $T X$ does. In other words, $Y$ is almost contact if $X$ is almost symplectic.

Now take $X$ to be closed almost symplectic with $\pi_{1}(X)=\Gamma$. After replacing $X$ by $X \# \overline{\mathbb{C} P^{n}}$ if necessary, we may assume $\mathbb{Z} \subset \pi_{2}(X)$. Choose the circle bundle $Y$ to restrict to have degree one on a 2 -sphere generating $\mathbb{Z}$. Then in the exact sequence of homotopy groups induced by $\pi$,

$$
\pi_{2}(X) \longrightarrow \pi_{1}\left(S^{1}\right) \longrightarrow \pi_{1}(Y) \longrightarrow \pi_{1}(X) \longrightarrow 1,
$$

the first map is surjective, so $\pi_{1}(Y)=\Gamma$.

\section{References}

1. W. M. BoothвY and H. C. WANG, 'On contact manifolds', Ann. of Math. 68 (1958) 721-734.

Received 31 July 1991.

1991 Mathematics Subject Classification 53C15, 57M05, 57R15.

Bull. London Math. Soc. 24 (1992) 493-494 
2. D. Koтschick, 'All fundamental groups are almost complex', Bull. London Math. Soc. 24 (1992) 377-378.

3. J. Martinet, 'Formes de contact sur les variétés de dimension 3', Proceedings of Liverpool Singularities Symposium II, Lecture Notes in Math. 209 (Springer, Berlin, 1971).

\author{
Mathematisches Institut \\ Universität Basel \\ Rheinsprung 21 \\ 4051 Basel \\ Switzerland
}

